\title{
Kybernetika
}

\section{Ton Geerts}

Free end-point linear-quadratic control subject to implicit continuous- time systems: Necessary and sufficient conditions for solvability

Kybernetika, Vol. 29 (1993), No. 5, 431--438

Persistent URL: http://dml.cz/dmlcz/124543

\section{Terms of use:}

(C) Institute of Information Theory and Automation AS CR, 1993

Institute of Mathematics of the Academy of Sciences of the Czech Republic provides access to digitized documents strictly for personal use. Each copy of any part of this document must contain these Terms of use.

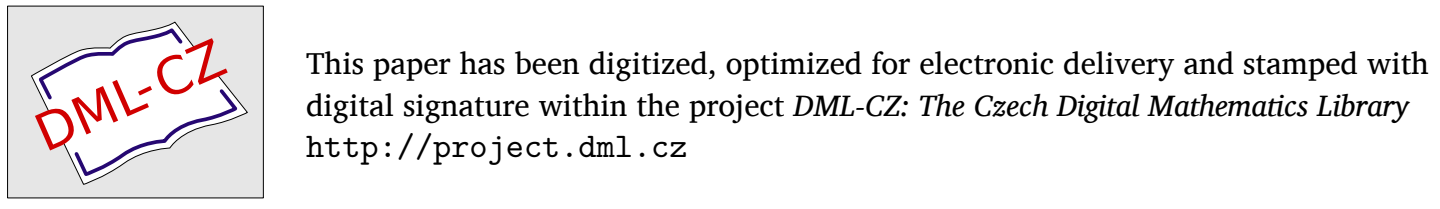




\title{
FREE END-POINT LINEAR-QUADRATIC CONTROL SUBJECT TO IMPLICIT CONTINUOUS-TIME SYSTEMS: NECESSARY AND SUFFICIENT CONDITIONS FOR SOLVABILITY
}

\author{
Ton Geerts ${ }^{1}$
}

For an implicit continuous-time system with arbitrary constant coefficients we derive necessary and sufficient conditions for solvability of the associated free end-point linearquadratic optimal control problem. In particular, this problem turns out to be solvable if and only if the underlying system is output stabilizable, as is the case for a standard system.

\section{INTRODUCTION AND PRELIMINARIES}

Given the implicit continuous-time system $\Sigma$ :

$$
\begin{aligned}
& E \dot{x}(t)=A x(t)+B u(t), \\
& y(t)=C x(t)+D u(t),
\end{aligned}
$$

with $u(t) \in \mathbb{R}^{m}, x(t) \in \mathbb{R}^{n}, y(t) \in \mathbb{R}^{r}$ for all $t \in \mathbb{R}^{+}:=[0, \infty)$. Let $k$ denote the number of equations in (1.1a) and let $e=\operatorname{rank}(E)$. All matrices involved are real-valued and constant. We may, and hence will, assume that $[E A B]$ is of full row rank. If $E$ is invertible, then the solutions of (1.1a) are

$$
x(t)=\exp \left(E^{-1} A t\right) x_{0}+\int_{0}^{t} \exp \left(E^{-1} A(t-\tau)\right) E^{-1} B u(\tau) \mathrm{d} \tau
$$

( $x_{0} \in \mathbb{R}^{n}$ arbitrary) and hence every $x_{0}$ is consistent, i.e., for every $x_{0},(1.1 \mathrm{a})$ has a solution $x$ with $x\left(0^{+}\right)=x_{0}$. If $E$ is not invertible, however, this need not be the case and inconsistent initial conditions may give rise to impulsive solutions of (1.1a), see e.g. [12], [2]. The most natural way to deal with such phenomena is the use of distributions [11], as was done earlier in e.g. [2]. Instead of (1.1), we will consider its distributional interpretation:

\footnotetext{
${ }^{1}$ Supported by the Dutch organization for scientific research (N.W.o.).
} 


$$
\begin{aligned}
& E \delta^{(1)} * x=A x+B u+E x_{0} \delta, \\
& y=C x+D u,
\end{aligned}
$$

where $\delta, \delta^{(1)}$ denote the Dirac distribution and its distributional derivative, respectively, $*$ stands for convolution of distributions, $x_{0} \in \mathbb{R}^{n}$, arbitrary. Moreover, $u \in \mathcal{C}_{\text {imp }}^{m}$, the $m$-vector version of $\mathcal{C}_{\text {imp }}$, the commutative algebra (over $\mathbb{R}$ ) of impulsive-smooth distributions [10, Def. 3.1], [9]. A distribution is impulsivesmooth if it can be decomposed (uniquely) in an impulse (any linear combination of $\delta$ and its derivatives $\delta^{(i)}, i \geq 1$ ) and a smooth distribution. A distribution is called smooth if it corresponds to a function that is smooth on $\mathbb{R}^{+}$and zero elsewhere. Let $\mathcal{C}_{\mathrm{sm}}$ denote the subalgebra of smooth distributions. The distributional derivative of $u \in \mathcal{C}_{\mathrm{sm}}, u^{(1)}=\delta^{(1)} * u$, equals $\dot{u}+u\left(0^{+}\right) \delta$, where $\dot{u} \in \mathcal{C}_{\mathrm{sm}}$ denotes the ordinary derivative of $u$ on $\mathbb{R}^{+}$. Example: Let $u \in \mathcal{C}_{\mathrm{sm}}$ correspond to $2 \exp (t)$ on $\mathbb{R}^{+}$. Then $u^{(1)}=\dot{u}+2 \delta$. For more details on $\mathcal{C}_{\text {imp }}$, see [9]-[10], also [6]-[8]; because of its nice properties we can keep our treatment fully algebraic. It can be readily shown that, for every real-valued square matrix $H,\left(I \delta^{(1)}-H \delta\right)$ is invertible (w. r. t. convolution); its inverse corresponds to $\exp (H t)$ on $\mathbb{R}^{+}$. Hence the solutions of (1.3a) reduce to the ordinary ones $((1.2))$ if $E$ is invertible and $u \in \mathcal{C}_{\mathrm{sm}}^{m}$; for every pair $\left(x_{0}, u\right),(1.3 \mathrm{a})$ has exactly one solution. Also, note that (1.3a) reduces to (1.1a) if $u$ and $x$ are smooth. In general, however, the solution set

$$
S\left(x_{0}, u\right)=\left\{x \in \mathcal{C}_{\mathrm{imp}}^{\mathrm{n}} \mid\left[E \delta^{(1)}-A \delta\right] * x=B u+E x_{0} \delta\right\},
$$

may be empty or contain infinitely many elements, see [6]. We are ready for the definition of the free end-point linear-quadratic control problem subject to (1.3).

(LQCP) $^{-}:$For all $x_{0}$, determine

$$
J^{-}\left(x_{0}\right):=\inf \left\{\int_{0}^{\infty} y^{\prime} y \mathrm{~d} t \mid u \in \mathcal{C}_{\mathrm{sm}}^{m}, x \in S\left(x_{0}, u\right) \cap \mathcal{C}_{\mathrm{sm}}^{n}\right\}
$$

and if, for every $x_{0}, J^{-}\left(x_{0}\right)<\infty$, then compute (if possible) optimal controls $\bar{u} \in \mathcal{C}_{\mathrm{sm}}^{m}$ and associated optimal state trajectories $\bar{x} \in S\left(x_{0}, \bar{u}\right)$. The problem (LQCP)- is solvable if both requirements are met.

In the sequel we will need several subspaces of interest. Let

$$
\begin{aligned}
& \mathcal{S}(\Sigma):=\left\{x_{0} \in \mathbb{R}^{n} \mid \exists u \in \mathcal{C}_{\mathrm{sm}}^{m} \exists x \in S\left(x_{0}, u\right) \cap \mathcal{C}_{\mathrm{sm}}^{n}: \lim _{t \rightarrow \infty}\left[\begin{array}{c}
u(t) \\
x(t)
\end{array}\right]=0\right\}, \\
& \mathcal{V}_{C}(\Sigma):=\left\{x_{0} \in \mathbb{R}^{n} \mid \exists u \in \mathcal{C}_{\mathrm{sm}}^{m} \exists x \in S\left(x_{0}, u\right) \cap \mathcal{C}_{\mathrm{sm}}^{n}: y=0, x\left(0^{+}\right)=x_{0}\right\} \\
& \mathcal{O}(\Sigma):=\left\{x_{0} \in \mathbb{R}^{n} \mid \exists u \in \mathcal{C}_{\mathrm{sm}}^{m} \exists x \in S\left(x_{0}, u\right) \cap \mathcal{C}_{\mathrm{sm}}^{n}: \lim _{t \rightarrow \infty} y(t)=0\right\}
\end{aligned}
$$

and let $\mathcal{S}_{B}(\Sigma), \mathcal{O}_{B}(\Sigma)$ denote those subspaces of $\mathcal{S}(\Sigma)$ and $\mathcal{O}(\Sigma)$, for which $u$ and $x$ in the respective definitions are of the Bohl type (a Bohl function is any linear combination of functions $\left.t^{k} \exp (\lambda t), k \geq 0\right)$. For $\mathcal{V}_{C}(\Sigma)$ we have the following result. 
Proposition 1.1. [7, Prop. 3.5, Theorem 3.6]. $\mathcal{V}_{C}(\Sigma)$ is the largest subspace $\mathcal{L} \subset \mathbb{R}^{n}$ for which there exists a matrix $F \in \mathbb{R}^{m \times n}$ such that $(A+B F) \mathcal{L} \subset$ $E \mathcal{L},(C+D F) \mathcal{L}=0$.

If, moreover,

$$
\mathcal{V}(\Sigma):=\left\{x_{0} \in \mathbb{R}^{n} \mid \exists u \in \mathcal{C}_{\mathrm{sm}}^{m} \exists x \in S\left(x_{0}, u\right) \cap \mathcal{C}_{\mathrm{sm}}^{n}: y=0\right\},
$$

then [7, Prop. 3.4] tells us that

$$
\mathcal{V}(\Sigma)=\mathcal{V}_{C}(\Sigma)+\operatorname{ker}(E)
$$

In [10], [7] a point $x_{0} \in \mathcal{V}(\Sigma)$ is called weakly unobservable; we establish that all points in $\mathcal{V}_{C}(\Sigma)$ are also consistent. Let, for any subspace $T$ and $\eta$ any complex row vector of compatible size, $\eta T$ stand for $\{\eta t \mid t \in T\}$. The next result is stated in [3].

Proposition 1.2. Let $E$ be invertible. Then $\mathcal{S}(\Sigma)+\mathcal{V}(\Sigma)=\mathcal{O}(\Sigma)=\left\{x_{0} \in\right.$ $\left.\mathbb{R}^{n} \mid J^{-}\left(x_{0}\right)<\infty\right\}, \mathcal{O}_{B}(\Sigma)=\mathcal{O}(\Sigma), \mathcal{S}_{B}(\Sigma)=\mathcal{S}(\Sigma)$ and $\mathcal{O}(\Sigma)=\mathbb{R}^{n}$ if and only if, for all $\lambda \in \mathrm{C}$ with $\operatorname{Re}(\lambda) \geq 0$,

$$
\eta[\lambda E-A,-B]=0 \text { and } \eta E \mathcal{V}(\Sigma)=0 \text { only if } \eta=0 .
$$

If in Proposition 1.2, $C=I$ and $D=0$, then $\mathcal{V}(\Sigma)=0$ and we reobtain the wellknown statement that $\mathcal{S}(\Sigma)=\mathbb{R}^{n}$ if and only if $\Sigma$ is (state) stabilizable. We will say that $\Sigma$ is output stabilizable if $\mathcal{O}(\Sigma)=\mathbb{R}^{n}$.

Now, we consider $\Sigma$ with arbitrary $E$. From [6, Theorem 4.5] we borrow

\section{Proposition 1.3.}

$$
\begin{aligned}
& \forall x_{0} \in \mathbb{R}^{n} \exists u \in \mathcal{C}_{\mathrm{sm}}^{m} \exists x \in S\left(x_{0}, u\right) \cap \mathcal{C}_{\mathrm{sm}}^{\mathrm{n}} \Longleftrightarrow \\
& \operatorname{im}(E)+\operatorname{im}(B)+A(\operatorname{ker}(E))=\mathbb{R}^{k}
\end{aligned}
$$

\section{MAIN RESULTS}

Without loss of generality, we may rewrite $\Sigma$ in the form

$$
\begin{aligned}
& {\left[\begin{array}{ll}
I & 0 \\
0 & 0
\end{array}\right] \delta^{(1)} *\left[\begin{array}{l}
x_{1} \\
x_{2}
\end{array}\right]=\left[\begin{array}{ll}
A_{11} & A_{12} \\
A_{21} & A_{22}
\end{array}\right]\left[\begin{array}{l}
x_{1} \\
x_{2}
\end{array}\right]+\left[\begin{array}{l}
B_{1} \\
B_{2}
\end{array}\right] u+\left[\begin{array}{ll}
I & 0 \\
0 & 0
\end{array}\right]\left[\begin{array}{l}
x_{01} \\
x_{02}
\end{array}\right] \delta} \\
& y=\left[C_{1} C_{2}\right]\left[\begin{array}{l}
x_{1} \\
x_{2}
\end{array}\right]+D u
\end{aligned}
$$


Assume that (1.10) is satisfied, i.e., that $\left[\begin{array}{ll}A_{22} & B_{2}\end{array}\right]$ is of full row rank. Let $T=$ $\left[\begin{array}{l}T_{1} \\ T_{2}\end{array}\right] \in \mathbb{R}^{(n+m-e) \times(n+m-k)}$, of full column rank, be such that $\left[A_{22} B_{2}\right] T=0$. Set $N:=A_{22} A_{22}^{\prime}+B_{2} B_{2}^{\prime}>0, L:=T^{\prime} T>0$. Then

$$
Q:=\left[\begin{array}{ll}
A_{22}^{\prime} & T_{1} \\
B_{2}^{\prime} & T_{2}
\end{array}\right] \text { is invertible, } Q^{-1}=\left[\begin{array}{lr}
N^{-1} & 0 \\
0 & L^{-1}
\end{array}\right] Q^{\prime} .
$$

If $\bar{\Sigma}$ denotes the standard system

$$
\begin{aligned}
& \delta^{(1)} * z=\bar{A} z+\bar{B} v+z_{0} \delta, \\
& w=\bar{C} z+\bar{D} v
\end{aligned}
$$

with

$$
\begin{aligned}
& \bar{A}:=A_{11}-\left[\begin{array}{ll}
A_{12} & B_{1}
\end{array}\right]\left[\begin{array}{l}
A_{22}^{\prime} \\
B_{2}^{\prime}
\end{array}\right] N^{-1} A_{21}, \bar{B}:=\left[\begin{array}{ll}
A_{12} & B_{1}
\end{array}\right] T, \\
& \bar{C}:=C_{1}-\left[\begin{array}{ll}
C_{2} & D
\end{array}\right]\left[\begin{array}{l}
A_{22}^{\prime} \\
B_{2}^{\prime}
\end{array}\right] N^{-1} A_{21}, \bar{D}:=\left[\begin{array}{ll}
C_{2} & D
\end{array}\right] T,
\end{aligned}
$$

then it turns out that all solutions for (1.3) can be expressed in solutions for (2.3) and vice versa.

Theorem 2.1. Let $\left[\begin{array}{c}x_{01} \\ x_{02}\end{array}\right] \in \mathbb{R}^{n}, u \in \mathcal{C}_{\text {imp }}^{m}$ and $\left[\begin{array}{l}x_{1} \\ x_{2}\end{array}\right] \in S\left(\left[\begin{array}{l}x_{01} \\ x_{02}\end{array}\right], u\right)$. Then $x_{1}=z\left(x_{01}, v\right),\left[\begin{array}{l}x_{2} \\ u\end{array}\right]=\left[\begin{array}{l}A_{22}^{\prime} \\ B_{2}^{\prime}\end{array}\right] N^{-1}\left(-A_{21}\right)\left(z\left(x_{01}, v\right)\right)+T v$ with $v=L^{-1}\left[T_{1}^{\prime} x_{2}+\right.$ $\left.T_{2}^{\prime} u\right] \in \mathcal{C}_{\mathrm{imp}}^{n+m-k}$. Moreover, $y=w\left(x_{01}, v\right)$. Conversely, let $z_{0} \in \mathbb{R}^{e}, v \in \mathcal{C}_{\mathrm{imp}}^{n+m-k}$, and $z=z\left(z_{0}, v\right)$. Then $u=-B_{2}^{\prime} N^{-1} A_{21} z+T_{2} v \in \mathcal{C}_{\text {imp }}^{m}$ and, for all $x_{02},\left[\begin{array}{l}x_{1} \\ x_{2}\end{array}\right] \in$ $S\left(\left[\begin{array}{l}z_{0} \\ x_{02}\end{array}\right], u\right)$ with $x_{1}=z$ and $x_{2}=-A_{22}^{\prime} N^{-1} A_{21} z+T_{1} v$. In addition, $y=$ $w\left(z_{0}, v\right)$.

Proof. First half. If in (2.3a) with $z_{0}=x_{01}$ we insert $v$ as prescribed, then $\delta^{(1)} * z=\bar{A} z+\left[\begin{array}{ll}A_{12} & B_{1}\end{array}\right] Q\left[\begin{array}{lr}N^{-1} & 0 \\ 0 & L^{-1}\end{array}\right]\left\{Q^{\prime}\left[\begin{array}{l}x_{2} \\ u\end{array}\right]+\left[\begin{array}{c}A_{21} x_{1} \\ 0\end{array}\right]\right\}+x_{01} \delta=\bar{A} z+$ $\left[\begin{array}{ll}A_{12} & B_{1}\end{array}\right]\left[\begin{array}{l}x_{2} \\ u\end{array}\right]+\left(A_{11}-\bar{A}\right) x_{1}+x_{01} \delta=\bar{A} z+\left(\delta^{(1)} * x_{1}-A_{11} x_{1}-x_{01} \delta\right)+\left(A_{11}-\right.$ $\bar{A}) x_{1}+x_{01} \delta=\delta^{(1)} * x_{1}+\bar{A}\left(z-x_{1}\right)$, by $(2.1)-(2.2)$. Hence $\left[I_{e} \delta^{(1)}-\bar{A} \delta\right] *\left(z-x_{1}\right)=0$ and $z-x_{1}=0$. Since $\left[\begin{array}{l}x_{2} \\ u\end{array}\right]=Q Q^{-1}\left[\begin{array}{l}x_{2} \\ u\end{array}\right]=\left[\begin{array}{l}A_{22}^{\prime} \\ B_{2}^{\prime}\end{array}\right] N^{-1}\left(-A_{21} x_{1}\right)+T v$, the rest is clear. The second half is now trivial. 
Observe that if in (2.1), $e=k$ (i.e., $E$ is of full row rank), then $T$ is invertible and $\bar{A}=A_{11}, \bar{C}=C_{1}$ in (2.3). Here is our first main result.

Theorem 2.2. If the system (1.3) satisfies (1.10), then $\mathcal{S}(\Sigma)+\mathcal{V}(\Sigma)=\mathcal{O}(\Sigma)=$ $\left\{x_{0} \in \mathbb{R}^{n} \mid J^{-}\left(x_{0}\right)<\infty\right\}, \mathcal{S}_{B}(\Sigma)=\mathcal{S}(\Sigma)$ and $\mathcal{O}_{B}(\Sigma)=\mathcal{O}(\Sigma)$. Moreover, $(1.3)$ is output stabilizable if and only if (1.9)-(1.10) are satisfied.

Proof. Consider (2.1) - (2.3). Then $\left[\eta_{1} \eta_{2}\right]\left[\begin{array}{rrrr}\lambda I & -A_{11} & -A_{12} & -B_{1} \\ & -A_{21} & -A_{22} & -B_{2}\end{array}\right]=0$ if and only if $\eta_{1}\left[\lambda I_{e}-\bar{A},-\bar{B}\right]=0$ and $\eta_{2}$ equals $-\eta_{1}\left[\begin{array}{ll}A_{12} & B_{1}\end{array}\right]\left[\begin{array}{l}A_{22}^{\prime} \\ B_{2}^{\prime}\end{array}\right] N^{-1}$, for every $\lambda \in \mathrm{C}$. Since $\operatorname{ker}(E)$ is contained in all subspaces involved, both claims follows immediately from Propositions 1.2, 1.3 and Theorem 2.1.

Now, let us consider (LQCP)- ${ }^{-}$. By Theorem 2.2, it is obvious that output stabilizability is necessary for solvability. Output stabilizability turns out to be sufficient for solvability as well.

Theorem 2.3. For every $x_{0} \in \mathbb{R}^{n}, J^{-}\left(x_{0}\right)<\infty$ if and only if the system (1.3) is output stabilizable. Assume this to be the case. Then there exists a unique real symmetric matrix $P^{-} \geq 0$, with $\operatorname{ker}(E) \subset \operatorname{ker}\left(P^{-}\right)$, such that, for all $x_{0}, J^{-}\left(x_{0}\right)=$ $x_{0}^{\prime} P^{-} x_{0}$. If

$$
\operatorname{ker}\left(\left[\begin{array}{ll}
E & 0 \\
C & D
\end{array}\right]\right) \cap[A B]^{-1} \operatorname{im}(E)=0
$$

then for every $x_{0}$ there exists a unique optimal control $\bar{u}$ and a unique optimal state trajectory $\bar{x} \in S\left(x_{0}, \bar{u}\right)$, both of the Bohl type. If (2.4) is not satisfied, then for every $x_{0}$ there exist $u \in \mathcal{C}_{\mathrm{imp}}^{m}$ and $x \in S\left(x_{0}, u\right)$ such that $y \in \mathcal{C}_{\mathrm{sm}}^{r}$ and $J^{-}\left(x_{0}\right)=\int_{0}^{\infty} y^{\prime} y \mathrm{~d} t$.

Proof. Assume that $\Sigma$ is output stabilizable. Consider the subsystem $\bar{\Sigma}(2.3)$, and let $\bar{J}^{-}\left(z_{0}\right):=\inf \left\{\int_{0}^{\infty} w^{\prime} w \mathrm{~d} t \mid v \in \mathcal{C}_{\mathrm{sm}}^{n+m-k}\right\}$. It follows from Theorem 2.1 that, for every $z_{0} \in \mathbb{R}^{e}, \bar{J}^{-}\left(z_{0}\right)<\infty$ if and only if, for every $x_{0} \in \mathbb{R}^{n}, J^{-}\left(x_{0}\right)<\infty$. Hence, by Theorem $2.2, \bar{\Sigma}$ is output stabilizable. Then there exists a unique $\bar{P}^{-} \geq 0$ such that, for all $z_{0} \in \mathbb{R}^{e}, \bar{J}^{-}\left(z_{0}\right)=z_{0}^{\prime} \bar{P}^{-} z_{0}[3]-[4]$. Hence there exists a unique $P^{-} \geq 0$, with $\operatorname{ker}(E) \subset \operatorname{ker}\left(P^{-}\right)$, such that, for every $x_{0} \in \mathbb{R}^{n}, J^{-}\left(x_{0}\right)=x_{0}^{\prime} P^{-} x_{0}$. Next, for every $z_{0}$ there exist a unique input $v$ and (thus) a unique resulting state trajectory $z$, both of the Bohl type, such that $z_{0}^{\prime} \bar{P}^{-} z_{0}=\int_{0}^{\infty} w^{\prime} w \mathrm{~d} t$, if $\operatorname{ker}(\bar{D})=0$, i.e., if the LQCP without stability subject to $\vec{\Sigma}$ is regular [4]. If $\operatorname{ker}(\vec{D}) \neq 0$, i.e., if this LQCP is singular, then for every $z_{0}$ there exist $v \in \mathcal{C}_{\text {imp }}^{n+m-k}$ and $z \in \mathcal{C}_{\text {imp }}^{e}$ 
such that $z_{0}^{\prime} \bar{P}^{-} z_{0}=\int_{0}^{\infty} w^{\prime} w \mathrm{~d} t$ [13], [5]; however, in general these optimal controls and optimal state trajectories have nonzero impulsive components. Observe that, in terms of $(2.1)-(2.3), \operatorname{ker}(\bar{D})=0$ if and only if $\operatorname{ker}\left(\left[\begin{array}{ll}A_{22} & B_{2} \\ C_{2} & D\end{array}\right]\right)=0$, and it is clear that the latter condition is equivalent to (2.4). The proof is now completed by application of Theorem 2.1 .

The condition (2.4) can be interpreted as a system property for $\Sigma$. In [8, Theorem 3.2 ] it is proven that (2.4) holds if and only if

$$
y \in \mathcal{C}_{\mathrm{sm}}^{r} \Longleftrightarrow u \in \mathcal{C}_{\mathrm{sm}}^{m}, x \in S\left(x_{0}, u\right) \cap \mathcal{C}_{\mathrm{sm}}^{\mathrm{n}}
$$

In other words, (2.4) stands for the property that outputs for $\Sigma$ are functions only if the output generating controls and state trajectories are functions as well. Therefore (LQCP) ${ }^{-}$is called regular in [8] if (2.5) is satisfied; note that (2.4) reduces to $\operatorname{ker}(D)=0$ if $E$ is invertible. The linear-quadratic control problems considered in [1] - [2] are regular in the sense of (2.4), since it is assumed there that $\operatorname{ker}\left(\left[\begin{array}{ll}E & 0 \\ C & D\end{array}\right]\right)=0$. An example of a regular linear-quadratic problem for which $\operatorname{ker}\left(\left[\begin{array}{ll}E & 0 \\ C & D\end{array}\right]\right) \neq 0$ is given in [8].

Observe that Theorem 2.3 states the existence of the matrix $P^{-}$; an explicit characterization of $P^{-}$, generalizing results in [4]- [5], will be given elsewhere. To the best of our knowledge, Theorem 2.3 contains the first general statements on (possibly) singular linear-quadratic control subject to implicit systems. Also, unlike in [1]- [2], we allow the state trajectories to diverge.

We will conclude this short paper with a by-result on uniqueness of optimal controls and optimal state trajectories for (LQCP) ${ }^{-}$.

If $\Sigma$ is output stabilizable and (2.4) is not satisfied, then we may still assume $\left[\begin{array}{cc}E & 0 \\ A & B\end{array}\right]$

$A \begin{array}{ll}A & B\end{array}$ to be of full column rank. Let this be the case. Now the distributional optimal controls and state trajectories for (LQCP)- (see Theorem 2.3) are in general not unique. This follows from Theorem 2.1, since it is proven in [5] that optimal controls and state trajectories for (LQCP) - subject to a standard system $\Sigma$ are unique if and only if $\Sigma$ is left invertible [10, Theorem 3.26], i.e., if in (1.3) with $E$ invertible, $y=0$ and $x_{0}=0$ imply that $u=0$ (and hence also $x=0$ ). Moreover, the smooth parts of these unique optimal controls and state trajectories are of the Bohl type. 
Two different concepts for left-invertibility for implicit systems are given in [7]. There, a system (1.3) is defined left invertible in the strong sense if $x_{0}=0$ and $y=0$ imply that $u=0$ and $E x=0$ (and left invertible in the weak sense if merely $u=0$ ), see [7, Defs. 4.1, 4.10]. Under the above-mentioned rank condition, it is proven in [7, Corollary 4.15] that $\Sigma$ is left invertible in the strong sense if and only if $x_{0}=0, y=0$ imply that $u=0, x=0$. Hence, again by Theorem $2.1, \Sigma$ is left invertible in the strong sense if and only if (2.3) is left invertible in the sense of [10] and thus

Corollary 2.4. Let $\Sigma$ be output stabilizable and $\operatorname{ker}\left(\left[\begin{array}{cc}E & 0 \\ A & B \\ C & D\end{array}\right]\right)=0$. Then for every $x_{0}$ there exists exactly one (possibly distributional) $\ddot{u}$ and exactly one (possibly distributional) $\bar{x}$ such that $\bar{y} \in \mathcal{C}_{\mathrm{sm}}^{r}$ and $\int_{0}^{\infty-} \bar{y}^{\prime} \bar{y} \mathrm{~d} t=J^{-}\left(x_{0}\right)$ if and only if $\Sigma$ is left invertible in the strong sense. Moreover, if $\bar{u}_{2}, \bar{x}_{2}$ denote the smooth parts of $\bar{u}$ and $\bar{x}$, then $\bar{u}_{2}$ and $\bar{x}_{2}$ are of the Bohl type.

\section{ACKNOWLEDGEMENT}

I am indebted to CYGNE, Eindhoven, for constant encouragement and immaterial support.

(Received January 27, 1993.)

\section{REFERENCES}

[1] D. J. Bender and A. J. Laub: The linear-quadratic optimal regulator for descriptor systems. IEEE Trans. Automat. Control AC-32 (1987), 672-688.

[2] D. Cobb: Descriptor variable systems and optimal state regulation. IEEE Trans. Automat. Control $A C-28$ (1983), 601-611.

[3] A.H.W. Geerts and M.L.J. Hautus: The output-stabilizable subspace and linear optimal control. In: Robust Control of Linear Systems and Nonlinear Control, Progress in Systems and Control Theory, Vol. 4, Birkhäuser, Boston 1990, pp. 113-120.

[4] T. Geerts: A necessary and sufficient condition for solvability of the linear-quadratic control problem without stability. Systems Control Lett. 11 (1988), 47-51.

[5] T. Geerts: All optimal controls for the singular linear-quadratic problem without stability; a new interpretation of the optimal cost. Linear Algebra Appl. 116 (1989), 135-181.

[6] T. Geerts: Solvability conditions, consistency and weak consistency for linear differential-algebraic equations and time-invariant singular systems: The general case. Linear Algebra Appl. 181 (1993), 111-130.

[7] T. Geerts: Invariant subspaces and invertibility properties for singular systems: The general case. Linear Algebra Appl. 183 (1993), 61-88.

[8] T. Geerts: Regularity and singularity in linear-quadratic control subject to implicit continuous-time systems. Circuits, Systems Signal Process., to appear. 
[9] M.L. J. Hautus. The formal Laplace transform for smooth linear systems. (Lecture Notes in Economics and Mathematical Systems 131.) Springer-Verlag, Berlin 1976, pp. $29-46$.

[10] M. L. J. Hautus and L. M. Silverman: System structure and singular control. Linear Algebra Appl. 50 (1983), 369-402.

[11] L. Schwartz: Théorie des Distributions. Hermann, Paris 1978.

[12] G.C. Verghese, B.C. Levy and T. Kailath: A generalized state-space for singular systems. IEEE Trans. Automat. Control AC-26 (1981), 811-831.

[13] J.C. Willems, A. Kitapci and L. M. Silverman: Singular optimal control: A geometric approach. SIAM J. Control Optim. 24 (1986), 323-337.

Dr. Ton Geerts, Tilburg University, Department of Econometrics, P. O. Box 90153, 5000 LE Tilburg. The Netherlands. 